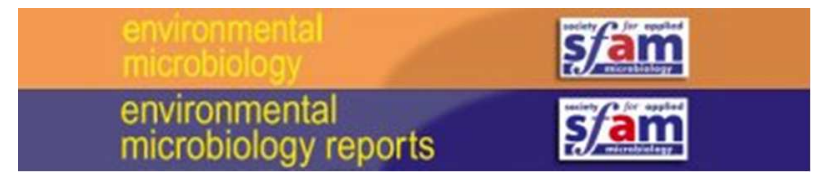

\title{
The effects of host age and spatial location on bacterial community composition in the English Oak tree (Quercus robur)
}

\begin{tabular}{|r|l|}
\hline Journal: & Environmental Microbiology and Environmental Microbiology Reports \\
\hline Manuscript ID & EMI-2016-0277.R1 \\
\hline Manuscript Type: & EMIR - Brief report \\
\hline Journal: & Environmental Microbiology Reports \\
\hline Complete List of Authors: & $\begin{array}{l}\text { Meaden, Sean; University of Exeter, College of Life and Environmental } \\
\text { Sciences } \\
\text { Metcalf, C.Jessica; Princeton University, Department of Ecology and } \\
\text { Evolutionary Biology; National Institute of Health, USA Fogarty } \\
\text { International Center } \\
\text { Koskella, Britt; University of California, Berkeley, Department of } \\
\text { Integrative Biology }\end{array}$ \\
\hline Keywords: & $\begin{array}{l}\text { microbial ecology, functional diversity, microbe: higher organism } \\
\text { interactions, microbial communities }\end{array}$ \\
\hline
\end{tabular}


1 The effects of host age and spatial location on bacterial community

2 composition in the English Oak tree (Quercus robur)

3

4 Meaden, S. ${ }^{1 *}$, Metcalf, C.J.E. ${ }^{2,3}$ and Koskella, B. ${ }^{4}$

5 Affiliations

6 1. College of Life and Environmental Sciences, University of Exeter, $7 \quad$ Penryn Campus, TR109FE, United Kingdom

2. Department of Ecology and Evolutionary Biology, Princeton University, Princeton, USA

3. Fogarty International Center, National Institute of Health, Bethesda, Maryland, USA

4. Department of Integrative Biology, University of California, Berkeley, 94720, USA

* Corresponding author: s.meaden@exeter.ac.uk 


\section{1}

32 2 3

\section{Summary}

Drivers of bacterial community assemblages associated with plants are diverse and include biotic factors, such as competitors and host traits, and abiotic factors, including environmental conditions and dispersal mechanisms. We examine the roles of spatial distribution and host size, as an approximation for age, in shaping the microbiome associated with Quercus robur woody tissue using culture-independent 16S rRNA gene amplicon sequencing. In addition to providing a baseline survey of the Q. robur microbiome, we screened for the pathogen of acute oak decline. Our results suggest that age is a predictor of bacterial community composition, demonstrating a surprising negative correlation between tree age and alpha diversity. We find no signature of dispersal limitation within the Wytham Woods plot sampled. Together, these results provide evidence for nichebased hypotheses of community assembly and the importance of tree age in bacterial community structure, as well as highlighting that caution must be applied when diagnosing dysbiosis in a long-lived plant host. (1)

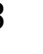

\section{0}

\section{1} 4 5 


\section{Introduction}

Many lines of evidence suggest that microbes are crucial for plant health and function (Kim et al., 2011; Berendsen et al., 2012), and yet we have a relatively poor understanding of which mechanisms shape the plantassociated microbial community or how this might in-turn influence host traits. Furthermore, although plant microbiome research has primarily focused on the below ground portion of the plant (the rhizosphere), knowledge of the phylloplane (the microbial composition of leaves) is increasing (Lindow and Brandl, 2003; Vorholt, 2012), demonstrating an equally important role in shaping plant phenotype. Still less is known regarding the microbial composition of other organs, with distinct communities reported across tissues within the host, often playing a more important role than biogeography (Ottesen et al., 2013; Leff et al., 2014; Coleman-Derr et al., 2016). For tree species in particular, the dermosphere (bark associated microbial community, (Lambais et al., 2014) may be particularly important given that bacterial pathogens often invade the host through wounds in the bark (Tattar, 2012; Misas-Villamil et al., 2013). This variation among tissues mirrors what is observed in other long-lived hosts, including humans, where data is most abundant; distinct bacterial communities have been isolated from different skin sites (Grice et al., 2009) and these differences appear stable over time (Costello et al., 2009). Such variation is also likely to exist across individual plant microbiomes given that they can be heritable (Peiffer et al., 2013), shaped by host genetics (Bodenhausen et al., 2014; Beckers et al., 2016), and play functional roles that include sensitizing the plant immune system (Pieterse et al., 2014). 
82 The root-associated microbiomes of healthy Arabidopsis plants are arguably

83 the best understood plant microbiome (Lundberg et al., 2012) with the

84 mechanisms behind host regulation recently coming to light (Lebeis et al.,

85 2015). However, many more non-model plant species have had their

86 microbiomes characterized. For example, a number of studies have explored

87 the nature of tree microbiomes, providing baseline taxonomic surveys and

88 assessing the drivers of community composition, typically contrasting host

89 traits with climatic or geographic variables. Many of these studies find a strong

90 effect of host phylogeny on the bacterial community, with a greater effect of

91 tree species than geographic distance, even across continents (Redford et al.,

92 2010; Lambais et al., 2014). Similarly, in a tropical environment in Malaysia,

93 Kim et al. (Kim et al., 2012) found a strong signal of host phylogeny on

94 bacterial community composition. Functional host traits such as growth rate

95 and leaf mass have also been demonstrated as key drivers of composition,

96 alongside phylogeny (Kembel et al., 2014). In contrast, Finkel et al. (Finkel et

97 al., 2012) found trees of the same species in a different desert locations host

98 distinct microbial communities. Given these conflicting results across the

99 scales examined, it is unclear whether phylloplane microbiomes are subject to

100 niche-based or neutral models of community assembly.

101

102 Specifically, the roles of dispersal and immigration, in combination with

103 ecological selection and drift (Vellend, 2010), have been the focus of a

104 number of theoretical models of community assembly, many of which are

105 applicable to microbes (Sloan et al., 2006; Nemergut et al., 2013). The niche 
106 assembly model states that the dispersal of bacteria is unhindered by physical

107 constraints, and all organisms can be found anywhere but it is the

108 environment which selects for their persistence (de Wit and Bouvier, 2006).

109 Conversely, the dispersal assembly hypothesis states that the biodiversity we

110 observe can largely be explained by stochastic local extinctions and dispersal-

111 limitation, typified by the idea of island biogeography (Hubbell, 2001; Volkov

112 et al., 2003). Whilst this is essentially the "niche vs. neutral" debate, Fierer

113 (Fierer, 2008) provides the nuances of the microbial context including the

114 much higher species richness and evenness, and the rapidity of species

115 turnover typical of most bacterial communities.

117 The English oak tree, Quercus robur, study system provides an opportunity to

118 test these competing hypotheses. If microbial community assembly is purely a

119 dispersal-driven process, we would predict a positive relationship between

120 tree age and diversity, as older organisms will have experienced more

121 colonization events. Such a positive relationship has been demonstrated for

122 trees and their plant epiphytes and lichens (Flores-Palacios and Garcia-

123 Franco, 2006; Johansson et al., 2007), but has not been shown before in tree-

124 associated bacterial communities. Alternatively, if the process is strictly niche-

125 driven, older trees could represent an alternative environment to smaller

126 trees, favoring proliferation of particular species but not necessarily harboring

127 a greater diversity.

129 As well as dispersal, host traits are likely to govern the microbes present.

130 Among the host factors known to influence microbial diversity, host age is 
131 often a key predictor. Data from humans suggests diversity consistently

132 increases with age from birth across populations (Yatsunenko et al., 2012). In

133 insects, honey bee queens undergo massive compositional shifts in their

134 microbiome as they age (Tarpy et al., 2015) and in a wild bird, Rissa

135 tridactyla, chicks harbor a greater diversity of bacteria than adults (van

136 Dongen et al., 2013). In plants, bacterial diversity can be highest on younger

137 leaves in lettuces (Dees et al., 2015), however the evidence is mixed as tree-

138 associated bacterial communities can be strongly influenced by season

139 (Peñuelas et al., 2012).

140

141 In this study we describe and explore the bacterial composition of Q.robur tree 142 cores in a well-studied UK forest, Wytham Woods, in order to answer three 143 key questions: firstly, what are the typical bacterial taxa associated with this

144 Woodland site; secondly, does geographic distance affect dispersal, such that

145 there is a spatial pattern of community composition and distance between

146 trees; and thirdly, is Q. robur host age or location important in structuring 147 bacterial communities. To answer these questions, we first describe the tree148 associated microbiota using amplicon sequencing of the 16S rDNA gene of 64

149 trees. Using a long-term woodland census we then assess correlations

150 between alpha and beta diversity and factors such as age and spatial

151 location. Additionally, we use these data to compare the predicted metabolic

152 functionality and screen our dataset for the pathogenic clade Brenneria, the

153 causative agent of acute oak decline, from which the UK Q. robur population

154 is currently experiencing an epidemic (Denman et al., 2012). This survey

155 presents a unique opportunity to assess the practicality of high throughput 
156 sequencing in environmental monitoring. Given the critical importance of

157 detecting and preventing the emergence of tree diseases before large-scale

158 spread, a better understanding of tree microbiomes offers additional value in

159 surveillance.

160

161

162

163

164

165

166

167

168

169

170

171

172

173

174 from the Brenneria genus have been isolated from Q. robur trees suffering

175 from AOD and it is likely that this species is the causal agent (Denman et al.,

176 2012). The disease has not yet been reported in Wytham Woods (Kirby et al.,

177 2014) so the absence of Brenneria species on healthy trees would buttress

178 the existing evidence that Brenneria is the primary pathogen. 
181 We sampled 64 Q. robur trees in a single hectare, collecting 192 samples in

182 over 3 days in September, 2013. Tree diameter at breast height (DBH) was

183 recorded as a proxy for tree age. This method is endorsed by the UK forestry

184 commission as a non-destructive mode of estimating tree age (Commission,

185 1998). Whilst comparisons among trees at different sites due to crowding may

186 be inaccurate, comparisons of the same species at the same site provides

187 reliable estimates of tree age. Further, using trees from our dataset that had

188 known planting dates, we observe a linear relationship between diameter and

189 age, reinforcing the view that $\mathrm{DBH}$ is a good proxy for age (SI, Figure 1).

191 Core tissue samples were obtained using the Trephor tool (Rossi et al., 2006),

192 allowing for three small (approximately $3 \mathrm{~cm}$ ) microcore samples to be taken

193 at breast height at three separate sites (North, Southwest, and Southeast).

194 The tool was sterilized and wiped thoroughly using $70 \%$ ethanol in between

195 each sample extraction. Samples were flash frozen in the field for

196 transportation back to the laboratory. Upon return to the laboratory, samples

197 were homogenized using a Fast-Prep 24 instrument (MP Biomedicals) for five

198 minutes with the addition of two $0.5 \mathrm{~cm}$ steel beads. Total DNA was then

199 extracted from the resulting homogenate using a Qiagen DNeasy Plant Mini

200 Kit, following the protocol provided. For amplification of the V4 region of the

201 16S rDNA gene, the universal primer set GTGCCAGCMGCCGCGGTAA (5' --

202 - 3') and GGACTACHVGGGTWTCTAAT (5' --- 3') was used.

203

204

Brenneria amplification 
205 To demonstrate that the primers used in our study could amplify Brenneria

206 goodwinii we cultured strain 931-23 (provided by R. Jackson, University of

207 Reading) and chose a random subset of 5 of the primers used to amplify the

208 V4 region to test for amplification in these positive controls.

209

210 Bioinformatic analysis

211 Illumina MiSeq 250bp paired-end reads were demultiplexed and de-barcoded

212 at the sequencing centre (Source Biosciences, Oxford). Sequences have

213 been deposited in the NCBI short-read archive (accession PRJNA 298668).

214 Quality filtering of reads was conducted using the Qiime (1.9.0) pipeline

215 (Caporaso et al., 2010). Reads were joined and filtered with the default

216 settings (Bokulich et al., 2013). Briefly, a maximum of 3 consecutive low

217 quality base calls was allowed before truncating the read, phred-score

218 threshold was set at 30 (which provides a 99.9\% accuracy of base call), $75 \%$

219 of the read was required to consist of high-quality, consecutive base call and

220 all reads with $\mathrm{N}$ character base calls were dropped. Open reference OTU

221 picking was conducted using the Uclust algorithm and the Silva $11116 \mathrm{~S}$

222 rDNA database at the 97\% identity level (Pruesse et al., 2007; Edgar, 2010).

223 Chimera removal was performed with Chimera Slayer (Haas et al., 2011);

224 OTUs present at abundances less than $0.005 \%$ of the dataset were removed

225 as were OTUs observed in only a single instance, as both are known to inflate

226 diversity estimates (Bokulich et al., 2013). Mitochondrial and chloroplast

227 sequences were also removed. This left a remaining dataset with a total of

2281013881 sequences spread across 115 samples, containing a median count

229 of 1830 sequences per sample (mean 8816 , length: $251.8 \mathrm{bp}$ ). Using the 
230 same trimmed sequence files, closed-reference OTU picking was performed

231 against the GreenGenes (1.5) (DeSantis et al., 2006) database (as required

232 by Picrust) using the uclust algorithm implemented in Qiime (1.9). Functional

233 predictions of observed taxa was made using the Picrust program (Langille et

234 al., 2013) using the Kegg orthology database (Kanehisa et al., 2014).

237 Statistical analysis

238 Rarefaction was performed for diversity analyses to a depth of 500 sequences

239 per sample. Whilst this is relatively low for microbiome studies, we aimed to

240 maintain high levels of biological replication at the cost of sampling depth

241 within individual samples. Pseudo $\mathrm{R}^{2}$ values were calculated using the

242 residual and null deviance from model outputs as described in Faraway

243 (2006). UniFrac scores were generated in Qiime and statistical analyses were

244 performed in R (R Core Team, 2015) using the packages 'vegan' (Oksanen et

245 al., 2016) and 'cluster' (Maechler et al., 2015).

246

\section{Results}

249 Baseline survey of the Quercus robur microbiome

250 The most abundant bacterial class observed within our samples was the

251 alphaproteobacteria, with a mean relative abundance of $26 \%$ (+/- 12 S.D.),

252 followed by the thermoleophilia with $22 \%$ (+/- 15 S.D.), and the

253 betaproteobacteria, contributing a mean of $13 \%$ (+/- 15 S.D.). Overall,

254 acidobacteria, actinobacteria and proteobacteria were the three most

255 abundant phyla, making up over $80 \%$ of OTUs (Figure 1). 
257 Age related decline in microbial diversity

258 We identified a weak negative correlation between tree size and species

259 richness (using observed OTUs) when controlling for uneven sampling of

260 individual trees $\left(G L M, F_{1,87}=4.13, p=0.0453\right.$, pseudo $\left.R^{2}=0.048\right)$ (Figure 2.).

261 Observed OTU count was used as the measure of species richness, however

262 the result was non-significant when Faith's phylogenetic distance or Chao 1

263 estimator (Chao et al., 2004) was used ( $p=0.12$ and 0.16 respectively). There

264 was no effect of sample orientation (cardinal direction), and this factor was

265 therefore excluded from the model during stepwise model simplification.

266 Interestingly, these correlations strengthened when sample size was

267 increased to 110 samples by using a lower rarefaction depth (100 sequences,

268 data not shown). A similar result was mirrored by beta-diversity, where tree

269 size was a significant predictor of microbial community composition using both

270 abundance weighted UniFrac scores (PERMANOVA, $F_{1,87}=4.63, p=0.0036$,

$271 \mathrm{R}^{2}=0.052$, permutations $\left.=9999\right)$ and unweighted UniFrac scores

272 (PERMANOVA, $F_{1,87}=2.93, p=0.027, R^{2}=0.033$, permutations $=9999$ ) when

273 tree ID was controlled for.

275 Taxa correlations

276 To investigate changes in composition further we performed Spearman rank

277 correlations against tree size for each OTU in the dataset, and found no 278 significant associations following correction for multiple testing. To further 279 assess whether there were higher taxonomic level associations between 280 specific bacterial clades and tree size we selected the three most abundant 
281 phyla. Collectively, the Proteobacteria, Actinobacteria and Acidobacteria

282 made up over $80 \%$ of our sequences. We found a significant decrease in the

283 relative abundance of Proteobacteria with tree size (Kendall's rank correlation,

$284 \mathrm{~T}=-0.22, \mathrm{z}=-3.39, \mathrm{p}=0.0007)$, a significant increase in the relative abundance

285 of Actinobacteria ( $\mathrm{T}=0.19, \mathrm{z}=3.04, \mathrm{p}=0.0023)$ and a non-significant decrease

286 in Acidobacteria ( $\mathrm{T}=-0.14, \mathrm{z}=-2.13, \mathrm{p}=0.033)$ following Bonferroni correction

287 (Figure 3).

288

289 Functional predictions

290 In order to predict how the function of communities associated with our Q.

291 robur trees changed as they aged we created a predicted metagenome using

292 the Picrust program (Langille et al., 2013). However, we found no correlation

293 between any of the predicted individual genes or functional pathways

294 associated with our observed microbiome and tree size, perhaps indicating

295 high functional redundancy of the more diverse microbiota of smaller trees.

297 Assessing spatial patterns

298 Finally, to look for patterns of biogeography, or dispersal limitation, we

299 performed Mantel correlations between a spatial matrix from the Euclidean

300 distances between trees and the UniFrac scores that measure bacterial

301 community composition. A correlation would be indicative that the spatial

302 distribution of trees does indeed affect the bacterial composition of the

303 community. There was no effect of abundance for either weighted (Mantel $r=$

$3040.0009, p=0.47$, permutations $=9999)$ or unweighted UniFrac scores $(r=0.002$,

$305 p=0.46$, permutations $=9999)$, suggesting an absence of dispersal limitation. 
Brenneria

308 Reassuringly, we found no sequences identified as Brenneria in our dataset

309 (prior to rarefaction), despite confirming that all our tested primers could

310 successfully amplify this species following culture in vitro.

\section{Discussion}

313 Our study of the bacterial microbiomes of 64 English oak trees (Quercus

314 robur) in a single woodland provides a number of insights into the drivers of

315 bacterial community structure and dispersal. Firstly, our census of the

316 microbiome of $Q$. robur tissue is consistent with a previous report that found

317 the same 3 most dominant phyla in the roots of oak trees: Actinobacter,

318 Proteobacteria and Acidobacter (Uroz et al., 2010). The high abundance of

319 Acidobacter is also consistent with other culture-independent studies of the

320 phyllospheric microbiota from tropical trees (Kim et al., 2012).

322 By comparing tree size with species richness, we found no sign of an increase

323 in bacterial diversity as trees age. This is of particular interest as it suggests

324 factors other than dispersal affect microbiome structure, as would be

325 expected by an increase in microbial diversity with growth as a result of

326 species accumulation. When observed OTUs was used as the measure of

327 alpha diversity we found a weak but significant decline in species richness

328 with tree age. Furthermore, negative correlations between tree age and

329 species richness were significant when the sample size was increased by

330 reducing rarefaction depth (and therefore excluding fewer samples). Detecting

331 subtle changes in species diversity require maximal statistical power, and 
332 there is clearly a trade-off between sampling depth and statistical power.

333 Exploring this trade-off in regard to microbial community sampling clearly

334 warrants further study as alternative approaches have yet to be widely

335 adopted (McMurdie and Holmes, 2014). Moreover, quantifying the shape of

336 the age-diversity relationship through the tree lifetime requires longitudinal

337 studies to build on cross-sectional studies like the data presented here. One

338 suggestion for observed age-related differences is variation in the chemical

339 and physiological state of the host tissue (van Dongen et al., 2013) and this

340 could be the case between younger and older Q.robur tree tissues.

343 A flat or negative correlation between tree age and bacterial alpha diversity

344 contrasts the positive association found between epiphytic plants and lichens

345 and tree host age (Flores-Palacios and Garcia-Franco, 2006; Johansson et

346 al., 2007) perhaps suggesting that bacteria are less dispersal-limited than

347 other tree-associated organisms. To explore these ideas further, and based

348 on the conflicting niche assembly and dispersal assembly hypotheses

349 (Hubbell, 2001; de Wit and Bouvier, 2006), we predicted that if microbiome

350 structure is purely a function of dispersal, such that communities are

351 assembled by stochastic dispersal events and local extinctions, we would find

352 a correlation between spatial distance among trees and community

353 dissimilarity scores (beta diversity). Conversely, if microbes have unlimited

354 dispersal within the forest, as is often assumed, one would expect no

355 correlation with beta diversity. Our results suggest that latter models are most

356 informative, whereby we find no signature of dispersal limitation (i.e. the 
community composition of our samples are not influenced by the proximity of others). There is the potential for microbes to disperse at global scales (Morris et al., 2008), however evidence for true cosmopolitan distribution has been mixed to date (Caporaso et al., 2012; Finkel et al., 2012; Sul et al., 2013) and, as demonstrated by Bell (Bell, 2010) also in Wytham Woods, microbial dispersal limitation may be more important over short time scales.

We also found an increase in the relative abundance of Actinobacter and a decrease in Proteobacter and Acidobacter (although the latter was only nearing significance) with tree size. Mechanistically, it is hard to ascribe functions to whole phyla as they encompass a range of morphologies, metabolic diversity and pathogenicity (Dworkin et al., 2006). The Acidobacter are, however, reported to be slow growing with low metabolic rates (Ward et al., 2009), sometimes referred to as k-selection strategists due to their higher abundances in soils with lower resource availability (Fierer et al., 2008). Carbon mineralization rate can also be a good predictor of Acidobacter soil abundance, but how well these finding translates to an alternative niche, such as tree cores, remains unknown (Fierer et al., 2008). If this were the case in our system we would expect Acidobacter and Proteobacteria to be inversely correlated; but we find the opposite. Maignien et al. (Maignien et al., 2014) have also suggested that phyllosphere communities are first colonized by $r$ strategists (such as Acinetobacter and Pseudomonas). Moreover, when multiple OTUs of the same species are present in the source community, for example rainfall, only one becomes established in the phyllosphere 
382 community, indicative of niche competition (Maignien et al., 2014). Given that

383 the Acidobacter are consistently isolated at high relative abundances from soil

384 it seems likely that the soil is the major contributing source for the interior

385 microbiota of oak trees. Acidobacter have also been detected at high relative

386 abundances in the trunk of Gingko bilbao trees but not in the leaves of the

387 same trees, again suggesting soil derived rather than phyllospheric dispersal

388 (Leff et al., 2014). Whether this is through transport of microbes through the

389 phloem or a function of early, seedling colonization remains undetermined.

390 Interestingly, and as a word of caution, we identified the presence of Ralstonia

391 in our negative sequencing controls, which has been identified by Salter et al.

392 (Salter et al., 2014) as a common kit contaminant. However this group also

393 includes many plant pathogens and wouldn't be unexpected in our

394 environmental samples, highlighting the difficulty in identifying contaminant

395 sequences from environmental samples and the need for negative controls.

397 Whilst we have described a shift in bacterial community structure with age,

398 the correlations between specific taxa and age are only present at the phylum

399 level and not at the OTU level. The variability in genomic content, even

400 among closely related bacteria (Perna et al., 2001; Guidot et al., 2007), is

401 often used to justify a lack of ecological or metabolic similarity among hosts.

402 However there is evidence for functional convergence at higher taxonomic

403 ranks (Philippot et al., 2010), including trophic and biogeographic differences

404 (Fierer et al., 2008; Philippot et al., 2009). One mechanism for our observation

405 of size-based differences could be that the age of a plant is the most

406 important factor in determining its induced defenses (Quintero and Bowers, 
407 2011). Indeed, the complex interactions between host immune systems and

408 commensal bacteria are coming to light in different systems (Brestoff and

409 Artis, 2013; Franzenburg et al., 2013). For example, the presence of

410 commensal microbes is non-random in a tropical tree host and has been

411 demonstrated to prevent pathogen success, particularly in fungal endophytes

412 (Arnold et al., 2003).

413

414 Despite being present at low numbers, many species could collectively play a 415 role in microbial community function. To explore this idea further we used 416 metagenomic predictions based on our $16 \mathrm{~S}$ sequences to assess functional 417 diversity. Given that we found a significant shift in the microbial composition 418 (at the Phylum level) with tree age, we expected to find a similar effect of 419 functional traits. We found no such trend, as no individual genes or functional 420 pathways were over or under represented in older tree samples. This lack of

421 functional correlation, despite a taxonomic correlation implies a level of 422 redundancy in gene pathways among bacterial phyla, or lack of sensitivity in 423 the methods used to predict a metagenome. If the latter is true, and the

424 limitation is the quality of annotation in metagenomic databases then

425 ultimately, more metagenomic sequencing may not yield more insight into 426 community function.

428 A focus on Q. robur allows us to answer some important applied questions: A 429 reassuring outcome of this analysis was that we failed to identify a single 430 sequence from Brenneria species. The UK oak population is undergoing an 431 epidemic of acute oak decline (AOD) and the Brenneria clade of bacteria have 
432 been isolated from oaks experiencing the disease (Denman et al., 2012).

433 Koch's postulates have also been reported in the Spanish oak (Quercus ilex)

434 (Poza-Carrión et al., 2008). However acute oak decline was not found to be

435 present in Wytham in 2014 (Kirby et al., 2014) and our data supports that

436 conclusion. This further strengthens the inference that Brenneria is a

437 causative agent of the disease, as suggested by Denman et al. (Denman et

438 al., 2012). Our census provides a baseline of healthy microbial flora in UK Q.

439 robur and comparison with trees in diseased states is a crucial area for further

440 study. Additionally, the observed differences in microbiome among differently

441 aged trees provides a caution for defining tree microbiome health. The healthy

442 microbiome of a young tree may well appear similar as that of a dysbiotic

443 microbiome of an old tree. As such, when using microbiome studies in the

444 context of plant health, fair comparisons among plant demographics must be

445 made in order to make useful diagnoses.

446

447

448 Acknowledgements

449 This work was supported by a Royal Society Research Grant (to CJEM and

450 BK). SM was funded by a studentship at the University of Exeter, BK by a

451 NERC independent research fellowship (NE/K00879X/1), and CJEM by a

452 Royal Society Fellowship. The authors thank Dr. Rob Jackson at the

453 University of Reading for providing an isolate of Brenneria, Dr. Keith Kirby for

454 providing data on tree age and size and Dr. Konrad Paskiewicz as well as the

455 faculty and participants of the NERC-funded population genomics workshop

456 for training in bioinformatics analyses. 


\section{References}

459

460

461

462

463

464

465

466

467

468

469

470

471

472

473

474

475

476

477

478

479

480

481

482

483

484

485

486

487

488

489

490

491

492

493

Arnold, A.E., Mejía, L.C., Kyllo, D., Rojas, E.I., Maynard, Z., Robbins, N., and Herre, E.A. (2003) Fungal endophytes limit pathogen damage in a tropical tree. Proc. Natl. Acad. Sci. U. S. A. 100: 15649-54.

Beckers, B., Op De Beeck, M., Weyens, N., Van Acker, R., Van Montagu, M., Boerjan, W., and Vangronsveld, J. (2016) Lignin engineering in fieldgrown poplar trees affects the endosphere bacterial microbiome. Proc. Natl. Acad. Sci. U. S. A. 113: 2312-7.

Bell, T. (2010) Experimental tests of the bacterial distance-decay relationship. ISME J. 4: 1357-65.

Berendsen, R.L., Pieterse, C.M.J., and Bakker, P.A.H.M. (2012) The rhizosphere microbiome and plant health. Trends Plant Sci. 17: 478-86.

Bodenhausen, N., Bortfeld-Miller, M., Ackermann, M., and Vorholt, J.A. (2014) A synthetic community approach reveals plant genotypes affecting the phyllosphere microbiota. PLoS Genet. 10: e1004283.

Bokulich, N.A., Subramanian, S., Faith, J.J., Gevers, D., Gordon, J.I., Knight, R., et al. (2013) Quality-filtering vastly improves diversity estimates from Illumina amplicon sequencing. Nat. Methods 10: 57-9.

Brestoff, J.R. and Artis, D. (2013) Commensal bacteria at the interface of host metabolism and the immune system. Nat. Immunol. 14: 676-84.

Butt, N., Campbell, G., Malhi, Y., Morecroft, M., Fenn, K., and Thomas, M. (2009) Initial Results from Establishment of a Long-term Broadleaf Monitoring Plot at Wytham Woods, Oxford, UK.

Caporaso, J.G., Kuczynski, J., Stombaugh, J., Bittinger, K., Bushman, F.D., Costello, E.K., et al. (2010) QIIME allows analysis of high-throughput community sequencing data. Nat. Methods 7: 335-6.

Caporaso, J.G., Paszkiewicz, K., Field, D., Knight, R., and Gilbert, J.A. (2012) The Western English Channel contains a persistent microbial seed bank. ISME J. 6: 1089-93.

Chao, A., Chazdon, R.L., Colwell, R.K., and Shen, T.-J. (2004) A new statistical approach for assessing similarity of species composition with incidence and abundance data. Ecol. Lett. 8: 148-159.

Coleman-Derr, D., Desgarennes, D., Fonseca-Garcia, C., Gross, S., Clingenpeel, S., Woyke, T., et al. (2016) Plant compartment and biogeography affect microbiome composition in cultivated and native Agave species. New Phytol. 209: 798-811. 
UK Forestry Commision (1998) Estimating the Ade of Large and Veteran Trees in Britain. Forestry Commision Information Note

Costello, E.K., Lauber, C.L., Hamady, M., Fierer, N., Gordon, J.I., and Knight, R. (2009) Bacterial community variation in human body habitats across space and time. Science 326: 1694-7.

Dees, M.W., Lysøe, E., Nordskog, B., and Brurberg, M.B. (2015) Bacterial communities associated with surfaces of leafy greens: shift in composition and decrease in richness over time. Appl. Environ. Microbiol. 81: $1530-9$.

Denman, S., Brady, C., Kirk, S., Cleenwerck, I., Venter, S., Coutinho, T., and De Vos, P. (2012) Brenneria goodwinii sp. nov., associated with acute oak decline in the UK. Int. J. Syst. Evol. Microbiol. 62: 2451-6.

Denman, S. and Webber, J. (2009) Oak declines: new definitions and new episodes in Britain. Q. J. For. 103: 285-290.

DeSantis, T.Z., Hugenholtz, P., Larsen, N., Rojas, M., Brodie, E.L., Keller, K., et al. (2006) Greengenes, a chimera-checked 16S rRNA gene database and workbench compatible with ARB. Appl. Environ. Microbiol. 72: 506972.

Van Dongen, W.F., White, J., Brandl, H.B., Moodley, Y., Merkling, T., Leclaire, S., et al. (2013) Age-related differences in the cloacal microbiota of a wild bird species. BMC Ecol. 13: 11.

Dworkin, M., Falkow, S., Rosenberg, E., Schleifer, K.-H., and Stackebrandt, E. eds. (2006) The Prokaryotes Springer New York, New York, NY.

Edgar, R.C. (2010) Search and clustering orders of magnitude faster than BLAST. Bioinformatics 26: 2460-1.

Faraway, J. (2006) Extending the Linear Model in R. Taylor and Francis, Bota Roca FL.

Fierer, N. (2008) Microbial biogeography: patterns in microbial diversity across space and time. In, Zengler,K. (ed), Accessing Uncultivated Microorganisms: from the Environment to Organisms and Genomes and Back. ASM Press, Washington DC, pp. 95-115.

Fierer, N., Bradford, M.A., and Jackson, R.B. (2008) Toward an ecological classification of soil bacteria. Ecology. 88: 1354-1364.

Finkel, O.M., Burch, A.Y., Elad, T., Huse, S.M., Lindow, S.E., Post, A.F., and Belkin, S. (2012) Distance-decay relationships partially determine diversity patterns of phyllosphere bacteria on Tamarix trees across the Sonoran Desert [corrected]. Appl. Environ. Microbiol. 78: 6187-93. 
551

552

553

554

555

556

557

558

559

560

561

562

563

564

565

566

567 568

Flores-Palacios, A. and Garcia-Franco, J.G. (2006) The relationship between tree size and epiphyte species richness: testing four different hypotheses. J. Biogeogr. 33: 323-330.

Franzenburg, S., Walter, J., Künzel, S., Wang, J., Baines, J.F., Bosch, T.C.G., and Fraune, S. (2013) Distinct antimicrobial peptide expression determines host species-specific bacterial associations. Proc. Natt. Acad. Sci. U. S. A. 110: E3730-8.

Grice, E.A., Kong, H.H., Conlan, S., Deming, C.B., Davis, J., Young, A.C., et al. (2009) Topographical and temporal diversity of the human skin microbiome. Science 324: 1190-2.

Guidot, A., Prior, P., Schoenfeld, J., Carrère, S., Genin, S., and Boucher, C. (2007) Genomic structure and phylogeny of the plant pathogen Ralstonia solanacearum inferred from gene distribution analysis. J. Bacteriol. 189: $377-87$.

Haas, B.J., Gevers, D., Earl, A.M., Feldgarden, M., Ward, D. V, Giannoukos, G., et al. (2011) Chimeric 16S rRNA sequence formation and detection in Sanger and 454-pyrosequenced PCR amplicons. Genome Res. 21: 494504.

Hubbell, S.P. (2001) The Unified Neutral Theory of Biodiversity and Biogeography (MPB-32) Princeton University Press.

Hunter, M.D., Varley, G.C., and Gradwell, G.R. (1997) Estimating the relative roles of top-down and bottom-up forces on insect herbivore populations: A classic study revisited. Proc. Natl. Acad. Sci. 94: 9176-9181.

Johansson, P., Rydin, H., and Thor, G. (2007) Tree age relationships with epiphytic lichen diversity and lichen life history traits on ash in southern Sweden. Ecoscience 14: 81-91.

Kanehisa, M., Goto, S., Sato, Y., Kawashima, M., Furumichi, M., and Tanabe, M. (2014) Data, information, knowledge and principle: back to metabolism in KEGG. Nucleic Acids Res. 42: D199-205.

Kembel, S.W., O'Connor, T.K., Arnold, H.K., Hubbell, S.P., Wright, S.J., and Green, J.L. (2014) Relationships between phyllosphere bacterial communities and plant functional traits in a neotropical forest. Proc. Natl. Acad. Sci. 111: 13715-13720.

Kim, M., Singh, D., Lai-Hoe, A., Go, R., Abdul Rahim, R., Ainuddin, A.N., et al. (2012) Distinctive phyllosphere bacterial communities in tropical trees. Microb. Ecol. 63: 674-81.

Kim, Y.C., Leveau, J., McSpadden Gardener, B.B., Pierson, E.A., Pierson, L.S., and Ryu, C.-M. (2011) The multifactorial basis for plant health 
promotion by plant-associated bacteria. Appl. Environ. Microbiol. 77: 1548-55.

Kirby, K.J., Bazely, D.R., Goldberg, E.A., Hall, J.E., Isted, R., Perry, S.C., and Thomas, R.C. (2014) Changes in the tree and shrub layer of Wytham Woods (Southern England) 1974-2012: local and national trends compared. Forestry 87: 663-673.

Lambais, M.R., Lucheta, A.R., and Crowley, D.E. (2014) Bacterial community assemblages associated with the phyllosphere, dermosphere, and rhizosphere of tree species of the Atlantic forest are host taxon dependent. Microb. Ecol. 68: 567-74.

Langille, M.G.I., Zaneveld, J., Caporaso, J.G., McDonald, D., Knights, D., Reyes, J.A., et al. (2013) Predictive functional profiling of microbial communities using $16 \mathrm{~S}$ rRNA marker gene sequences. Nat. Biotechnol. 31: 814-21.

Lebeis, S.L., Paredes, S.H., Lundberg, D.S., Breakfield, N., Gehring, J., McDonald, M., et al. (2015) Salicylic acid modulates colonization of the root microbiome by specific bacterial taxa. Science (80-. ). 349: 860-4.

Leff, J.W., Del Tredici, P., Friedman, W.E., and Fierer, N. (2014) Spatial structuring of bacterial communities within individual Ginkgo biloba trees. Environ. Microbiol.

Lindow, S.E. and Brandl, M.T. (2003) Microbiology of the phyllosphere. Appl. Environ. Microbiol. 69: 1875-83.

Lundberg, D.S., Lebeis, S.L., Paredes, S.H., Yourstone, S., Gehring, J., Malfatti, S., et al. (2012) Defining the core Arabidopsis thaliana root microbiome. Nature 488: 86-90.

Maignien, L., DeForce, E.A., Chafee, M.E., Eren, A.M., and Simmons, S.L. (2014) Ecological succession and stochastic variation in the assembly of Arabidopsis thaliana phyllosphere communities. MBio 5: e00682-13.

McMurdie, P.J. and Holmes, S. Waste not, want not: Why rarefying microbiome data is inadmissable. PLoS Comput. Biol. 10: e1003531. doi:10.1371/journal.pcbi.1003531

Misas-Villamil, J.C., Kolodziejek, I., Crabill, E., Kaschani, F., Niessen, S., Shindo, T., et al. (2013) Pseudomonas syringae pv. syringae uses proteasome inhibitor syringolin A to colonize from wound infection sites. PLoS Pathog. 9: e1003281.

Morecroft, M.D., Stokes, V.J., and Morison, J.I.L. (2003) Seasonal changes in the photosynthetic capacity of canopy oak (Quercus robur) leaves: the impact of slow development on annual carbon uptake. Int. J. Biometeorol. 47: 221-6. 
608

609

610

611

612

613

614

615

616

617

618

619

620

621

622

623

624

625

626

627

628

629

630

631

632

633

634

635

636

637

638

639

640

641

642

643

644

645

Morris, C.E., Sands, D.C., Vinatzer, B.A., Glaux, C., Guilbaud, C., Buffière, A., et al. (2008) The life history of the plant pathogen Pseudomonas syringae is linked to the water cycle. ISME J. 2: 321-34.

Nemergut, D.R., Schmidt, S.K., Fukami, T., O’Neill, S.P., Bilinski, T.M., Stanish, L.F., et al. (2013) Patterns and processes of microbial community assembly. Microbiol. Mol. Biol. Rev. 77: 342-56.

Ottesen, A.R., González Peña, A., White, J.R., Pettengill, J.B., Li, C., Allard, S., et al. (2013) Baseline survey of the anatomical microbial ecology of an important food plant: Solanum lycopersicum (tomato). BMC Microbiol. 13: 114.

Peiffer, J.A., Spor, A., Koren, O., Jin, Z., Tringe, S.G., Dangl, J.L., et al. (2013) Diversity and heritability of the maize rhizosphere microbiome under field conditions. Proc. Natl. Acad. Sci. 110: 6548-6553.

Peñuelas, J., Rico, L., Ogaya, R., Jump, A.S., and Terradas, J. (2012) Summer season and long-term drought increase the richness of bacteria and fungi in the foliar phyllosphere of Quercus ilex in a mixed Mediterranean forest. Plant Biol. (Stuttg). 14: 565-75.

Perna, N.T., Plunkett, G., Burland, V., Mau, B., Glasner, J.D., Rose, D.J., et al. (2001) Genome sequence of enterohaemorrhagic Escherichia coli O157:H7. Nature 409: 529-33.

Philippot, L., Andersson, S.G.E., Battin, T.J., Prosser, J.I., Schimel, J.P., Whitman, W.B., and Hallin, S. (2010) The ecological coherence of high bacterial taxonomic ranks. Nat. Rev. Microbiol. 8: 523-9.

Philippot, L., Bru, D., Saby, N.P.A., Cuhel, J., Arrouays, D., Simek, M., and Hallin, S. (2009) Spatial patterns of bacterial taxa in nature reflect ecological traits of deep branches of the 16S rRNA bacterial tree. Environ. Microbiol. 11: 3096-104.

Pieterse, C.M.J., Zamioudis, C., Berendsen, R.L., Weller, D.M., Van Wees, S.C.M., and Bakker, P.A.H.M. (2014) Induced systemic resistance by beneficial microbes. Annu. Rev. Phytopathol. 52: 347-75.

Poza-Carrión, C., Aguilar, I., Gallego, F.J., Nuñez-Moreno, Y., Biosca, E.G., González, R., et al. (2008) Brenneria quercina and Serratia spp. isolated from Spanish oak trees: molecular characterization and development of PCR primers. Plant Pathol. 57: 308-319.

Pruesse, E., Quast, C., Knittel, K., Fuchs, B.M., Ludwig, W., Peplies, J., and Glöckner, F.O. (2007) SILVA: a comprehensive online resource for quality checked and aligned ribosomal RNA sequence data compatible with ARB. Nucleic Acids Res. 35: 7188-96. 
Quintero, C. and Bowers, M.D. (2011) Plant induced defenses depend more on plant age than previous history of damage: implications for plantherbivore interactions. J. Chem. Ecol. 37: 992-1001.

Redford, A.J., Bowers, R.M., Knight, R., Linhart, Y., and Fierer, N. (2010) The ecology of the phyllosphere: geographic and phylogenetic variability in the distribution of bacteria on tree leaves. Environ. Microbiol. 12: 28852893.

Rossi, S., Menardi, R., and Anfodillo, T. (2006) Trephor: A New Tool for Sampling Microcores from tree stems. IAWA J. 27: 89-97.

Salter, S.J., Cox, M.J., Turek, E.M., Calus, S.T., Cookson, W.O., Moffatt, M.F., et al. (2014) Reagent and laboratory contamination can critically impact sequence-based microbiome analyses. BMC Biol. 12: 87.

Sloan, W.T., Lunn, M., Woodcock, S., Head, I.M., Nee, S., and Curtis, T.P. (2006) Quantifying the roles of immigration and chance in shaping prokaryote community structure. Environ. Microbiol. 8: 732-40.

Sul, W.J., Oliver, T.A., Ducklow, H.W., Amaral-Zettler, L.A., and Sogin, M.L. (2013) Marine bacteria exhibit a bipolar distribution. Proc. Natl. Acad. Sci. U. S. A. 110: 2342-7.

Tarpy, D.R., Mattila, H.R., and Newton, I.L.G. (2015) Characterization of the honey bee microbiome throughout the queen-rearing process. Appl. Environ. Microbiol. 81: 3182-3191.

Tattar, T.A. (2012) Diseases of Shade Trees, Revised Edition Elsevier Science.

Uroz, S., Buée, M., Murat, C., Frey-Klett, P., and Martin, F. (2010) Pyrosequencing reveals a contrasted bacterial diversity between oak rhizosphere and surrounding soil. Environ. Microbiol. Rep. 2: 281-8.

Vellend, M. (2010) Conceptual Synthesis in Community Ecology. Q. Rev. Biol. 85: 183-206.

Volkov, I., Banavar, J.R., Hubbell, S.P., and Maritan, A. (2003) Neutral theory and relative species abundance in ecology. Nature 424: 1035-1037.

Vorholt, J.A. (2012) Microbial life in the phyllosphere. Nat. Rev. Microbiol. 10: 828-40.

Ward, N.L., Challacombe, J.F., Janssen, P.H., Henrissat, B., Coutinho, P.M., Wu, M., et al. (2009) Three genomes from the phylum Acidobacteria provide insight into the lifestyles of these microorganisms in soils. Appl. Environ. Microbiol. 75: 2046-56. 
682 De Wit, R. and Bouvier, T. (2006) "Everything is everywhere, but, the 683 684 environment selects"; what did Baas Becking and Beijerinck really say? Environ. Microbiol. 8: 755-8.

685 Yatsunenko, T., Rey, F.E., Manary, M.J., Trehan, I., Dominguez-Bello, M.G., 686 687 Contreras, M., et al. (2012) Human gut microbiome viewed across age and geography. Nature 486: 222-7.

688 


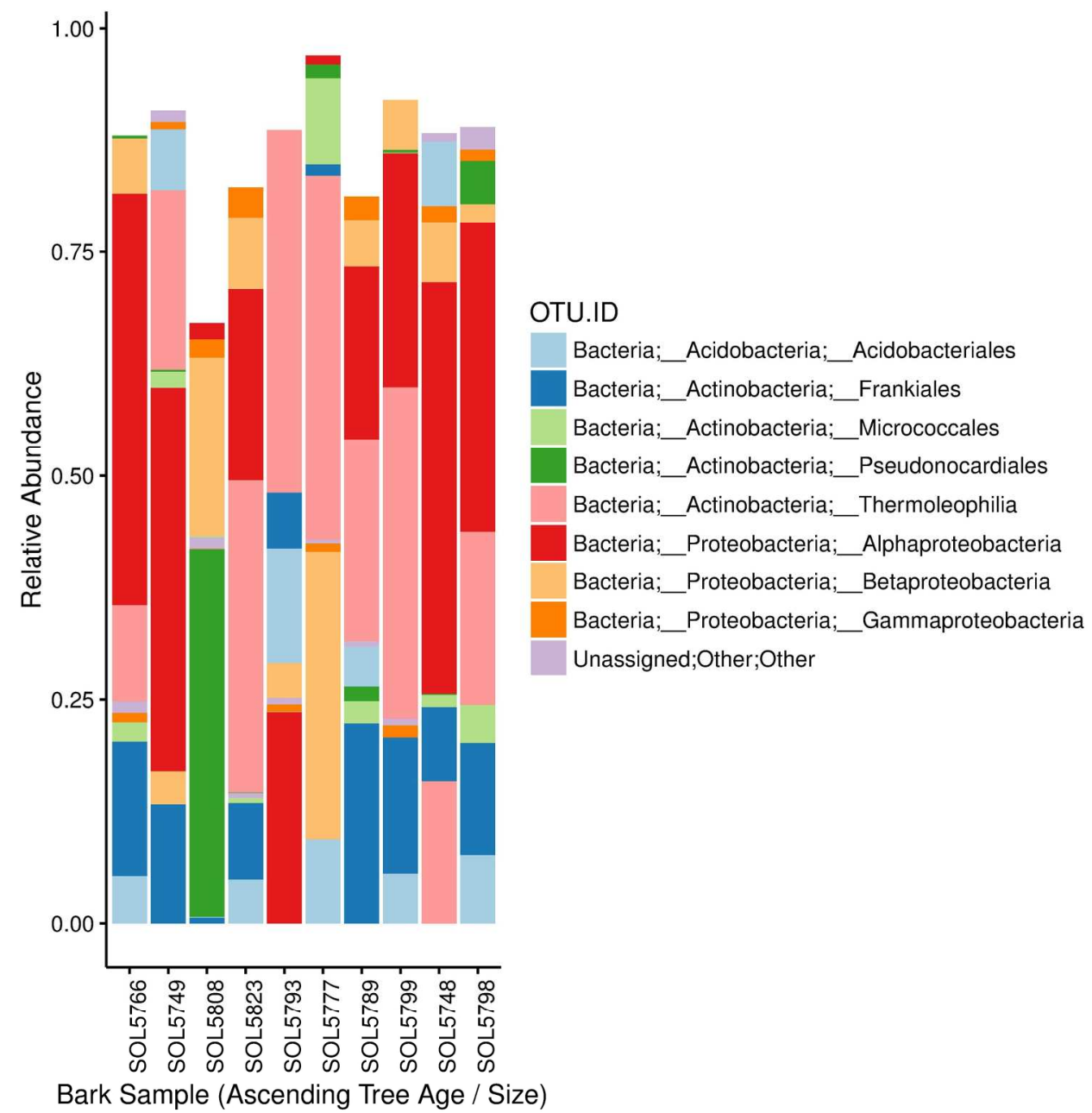

Randomly selected barplots showing the 9 most common bacterial taxa ordered by ascending tree age (from left to right). Alpha diversity decreases in older trees and there is much variation in beta-diversity. Only those taxa with a mean relative abundance greater than $2.5 \%$ across the entire dataset were retained for the figure for clarity. $177 \times 177 \mathrm{~mm}(300 \times 300$ DPI) 


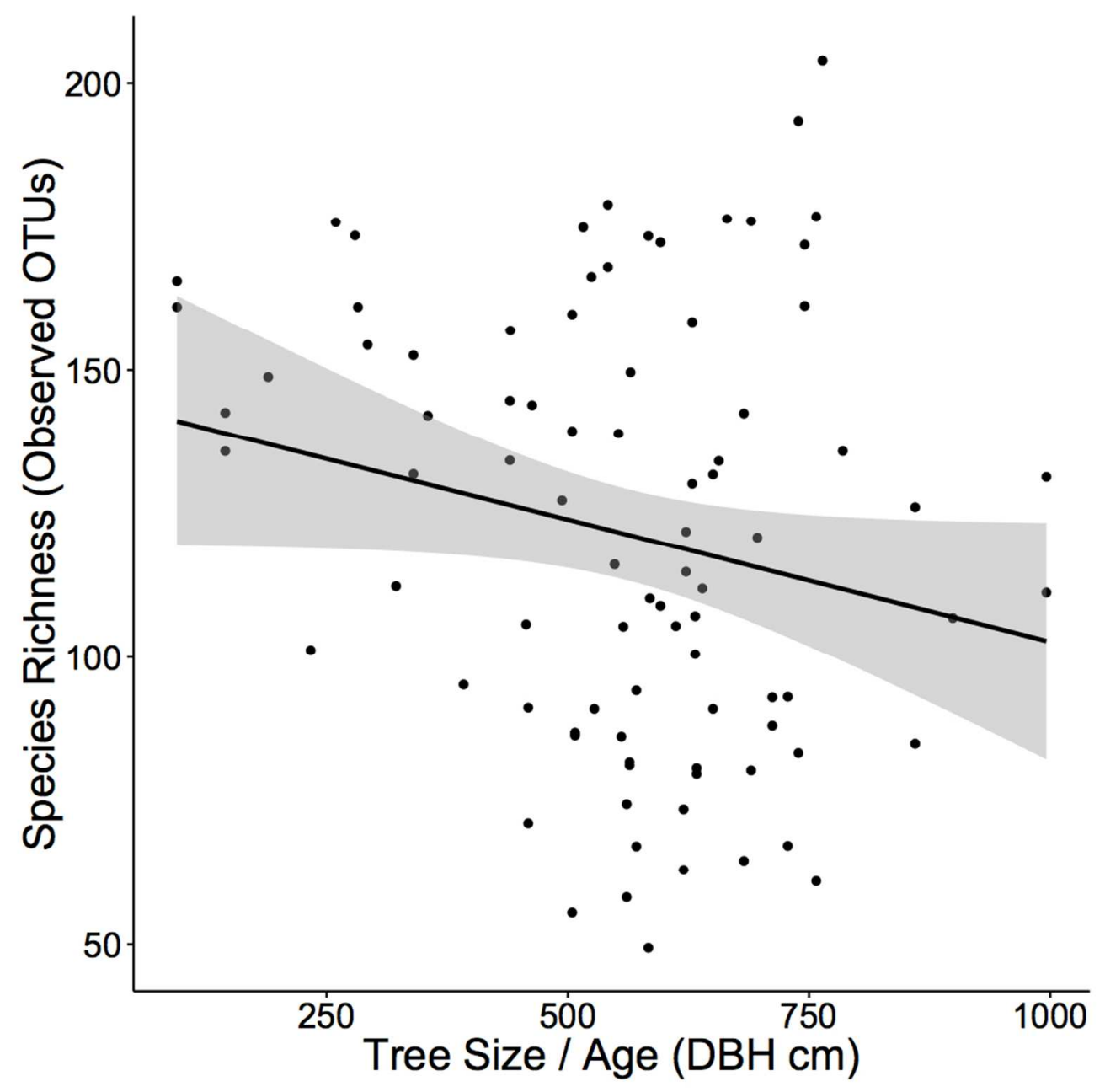

Age based decline in species richness based on species richness, as measured by observed OTUs, following rarefaction to a depth of 500 sequences per sample. GLM, $F 1,87=4.13, p=0.0453$. Intercept $=142$, slope $=-$ 0.045 .

$180 \times 177 \mathrm{~mm}(150 \times 150 \mathrm{DPI})$ 


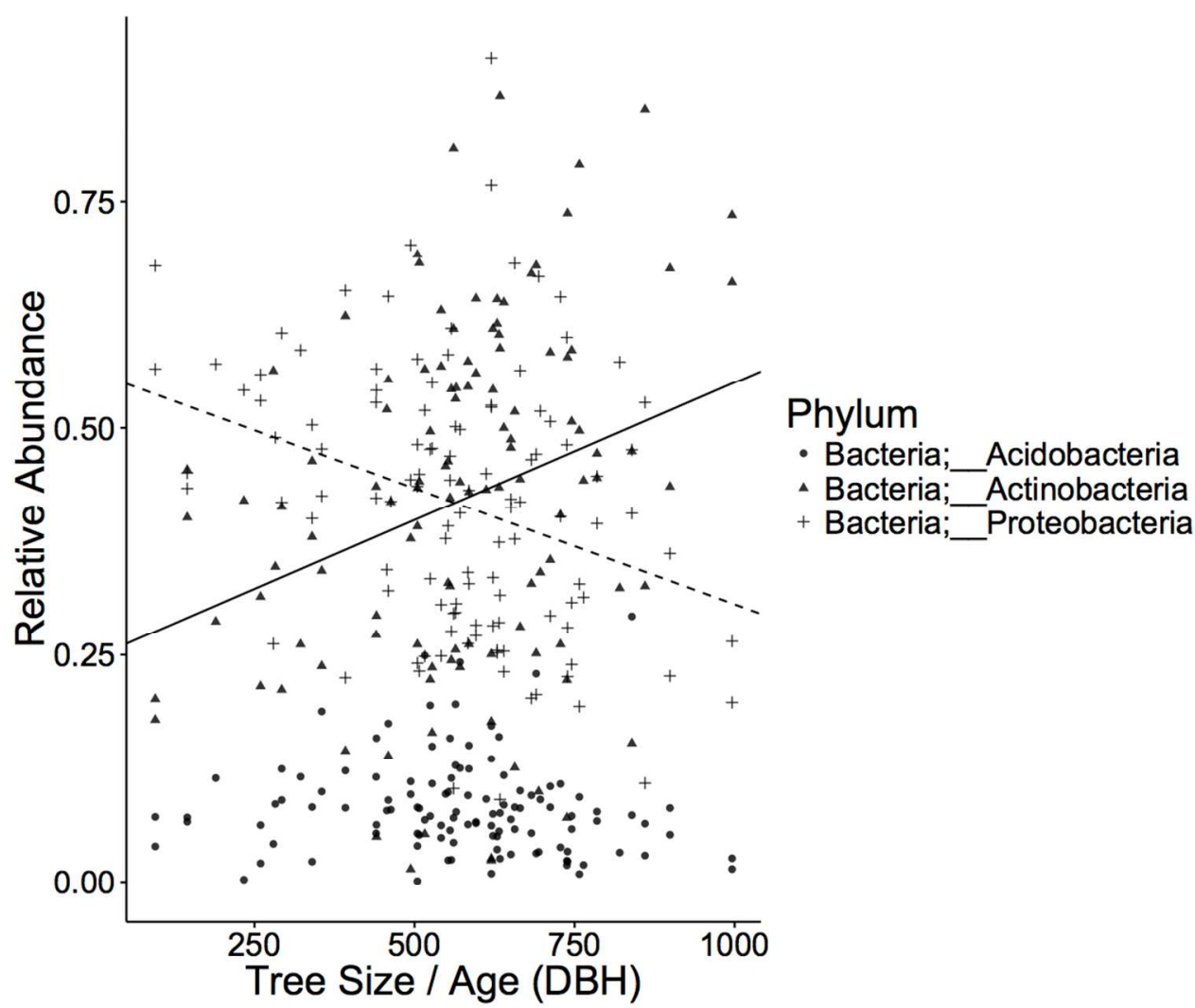

Taxa-specific correlations with Oak Tree age. The relative abundance of Actinobacteria increases with tree age (triangles, solid line, intercept $=0.25$, slope $=0.00030$ ) (Kendall's rank correlation, $\mathrm{T}=0.19, \mathrm{z}=3.04$, $\mathrm{p}=0.0023$ ), whilst the relative abundance of Proteobacteria declines (crosses, dashed line, intercept $=0.56$, slope $=-0.00026)(T=-0.22, z=-3.39, p=0.0007)$. The relative abundance of Acidobacteria also declines however this is non-significant after Bonferroni correction $(T=-0.14, z=-2.13, p=0.033)$. $217 \times 177 \mathrm{~mm}(150 \times 150 \mathrm{DPI})$ 


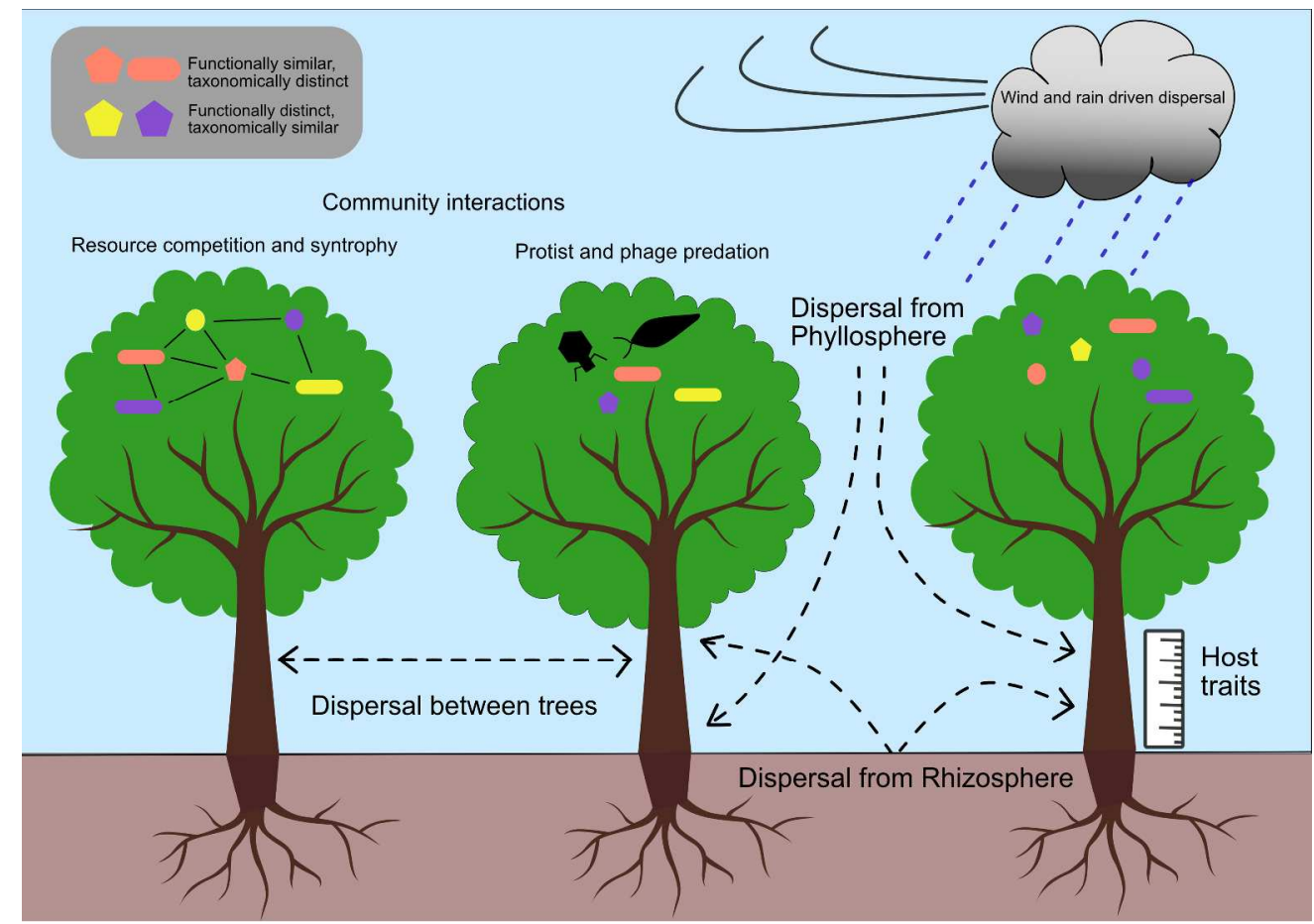

Conceptual diagram of potential drivers of bacterial community composition in our Oak tree system. Communities may be seeded from wind and rain driven dispersal, or colonize the plant directly from the soil during growth. Following initial colonization, the microbes must survive, and potentially thrive, in the observed niche. The niche is likely to be dictated by, among others, competition for host resources, predation and environmental conditions. 